\title{
Torus actions, Morse homology, and the Hilbert scheme of points on affine space
}

\author{
Burt Totaro
}

\begin{abstract}
We formulate a conjecture on actions of the multiplicative group in motivic homotopy theory. In short, if the multiplicative group $\mathbf{G}_{m}$ acts on a quasi-projective scheme $U$ such that $U$ is attracted as $t$ approaches 0 in $\mathbf{G}_{m}$ to a closed subset $Y$ in $U$, then the inclusion from $Y$ to $U$ should be an $\mathbf{A}^{1}$-homotopy equivalence. We prove several partial results. In particular, over the complex numbers, the inclusion is a homotopy equivalence on complex points. The proofs use an analog of Morse theory for singular varieties. Application: the Hilbert scheme of points on affine $n$-space is homotopy equivalent to the subspace consisting of schemes supported at the origin.
\end{abstract}

Keywords. Torus action, Morse homology, Hilbert scheme of points, motivic homotopy theory.

2020 Mathematics Subject Classification. 14L30, 14C05, 14F42, 55R80

\section{[Français]}

\section{Actions du tore, homologie de Morse et schéma de Hilbert des points de l'espace affine}

Résumé. Nous formulons une conjecture sur les actions du groupe multiplicatif en théorie de l'homotopie motivique. En bref, si le groupe multiplicatif $\mathbf{G}_{m}$ opère sur un schéma quasi-projectif $U$, de sorte que lorsque $t$ tend vers 0 dans $\mathbf{G}_{m}, U$ est contracté sur un sous-ensemble fermé $Y$, alors l'inclusion de $Y$ dans $U$ devrait être une $\mathbf{A}^{1}$-équivalence d'homotopie. Nous démontrons plusieurs résultats partiels. En particulier, sur le corps des complexes, l'inclusion est une équivalence d'homotopie sur les points complexes. Les preuves utilisent des analogues de la théorie de Morse pour des variétés singulières. Application : le schéma de Hilbert des points de l'espace affine de dimension $n$ est homotopiquement équivalent au sous-espace dont les points sont les schémas supportés à l'origine.

Received by the Editors on September 22, 2020, and in final form on May 25, 2021.

Accepted on June 23, 2021.

Burt Totaro

UCLA Mathematics Department, Box 951555, Los Angeles, CA 90095-1555

e-mail: totaro@math.ucla.edu

This work was supported by NSF grant DMS-1701237. I spoke about it in the Algebraic Geometry and Moduli Zoominar at ETH Zurich.

(C) by the author(s) 


\section{Contents}

1. Introduction. . . . . . . . . . . . . . . . . . . . . . . . . . . . . . . . 2

2. Main results, and a conjecture on $\mathbf{G}_{m}$-actions in motivic homotopy theory . . . . . . . 3

3. $\mathbf{G}_{m}$-actions and broken trajectories. . . . . . . . . . . . . . . . . . . . . . . 5

4. Openness . . . . . . . . . . . . . . . . . . . . . . . . . . . . . . . . . 6

5. Proof of Theorem 2.2 . . . . . . . . . . . . . . . . . . . . . . . . . . . . . . . . . . . . 7

6. The real case . . . . . . . . . . . . . . . . . . . . . . . . . . . . . . . 8

7. $\mathbf{A}^{1}$-connectedness of the Hilbert scheme

8. Conservativity for the motivic stable homotopy category . . . . . . . . . . . . . . 10

9. $\mathbf{G}_{m}$-actions on smooth varieties and motivic homotopy theory . . . . . . . . . . . 11

References. . . . . . . . . . . . . . . . . . . . . . . . . . . 13

\section{Introduction}

We formulate a conjecture on actions of the multiplicative group $\mathbf{G}_{m}$ in algebraic geometry. (Over the complex numbers, this group may be called $\mathbf{C}^{*}$.) In short, if $\mathbf{G}_{m}$ acts on a quasi-projective scheme $U$ which is attracted as $t \rightarrow 0$ in $\mathbf{G}_{m}$ to a closed subset $Y$ in $U$, then the inclusion $Y \rightarrow U$ should be an $\mathbf{A}^{1}$-homotopy equivalence (Conjecture 2.1). This is not obvious, in that the action of $\mathbf{G}_{m}$ on $U$ usually does not extend to a morphism $\mathbf{A}^{1} \times U \rightarrow U$; compare Figure 1. We show that the inclusion $Y \rightarrow U$ over the complex numbers is at least a homotopy equivalence in the classical topology (Theorem 2.2). This extends work of Hausel and Rodriguez-Villegas on the case where $U$ is smooth [HR15, Corollary 1.3.6]. We prove several other results in the direction of the conjecture, including a homotopy equivalence on real points (Theorem 6.1) and, when $U$ is smooth, an $\mathbf{A}^{1}$-homotopy equivalence after a suitable suspension (Theorem 9.1). The proofs use the ideas of Morse homology, translated into algebraic geometry (Proposition 3.1).

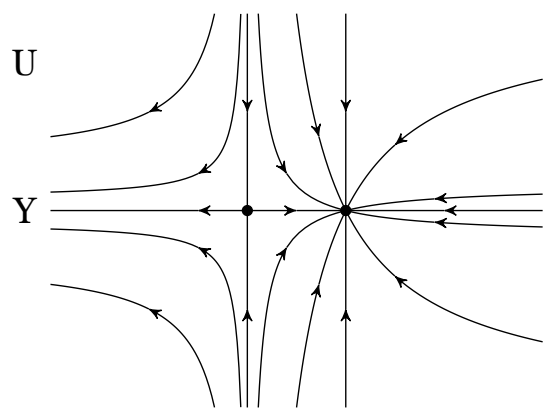

Figure 1. Example of a $T$-action on $U \cong \mathbf{P}^{1} \times \mathbf{A}^{1}, t\left(\left[x_{0}, x_{1}\right], y\right)=\left(\left[x_{0}, t x_{1}\right], t y\right)$, with $Y=\mathbf{P}^{1} \times 0$ shown as the horizontal line $\left(T=\mathbf{G}_{m}\right)$. The arrows point in the direction $t \rightarrow 0$. The fixed point set $Y^{T}$ consists of two points. 
We apply these results to the Hilbert scheme of points on affine space. The Hilbert scheme of points on an algebraic surface is smooth, and its Betti numbers were computed by Göttsche [Göt02]. The Hilbert scheme of points on a higher-dimensional variety, even affine space $\mathbf{A}^{n}$, is more mysterious. It has many irreducible components [Iar72], and for $n \geq 16$ its singularities satisfy Murphy's law up to retraction [Jel20]. Nonetheless, progress was recently made toward understanding the homotopy type (and even the $\mathbf{A}^{1}$-homotopy type) of $\mathrm{Hilb}_{d}\left(\mathbf{A}^{n}\right)$ for $n$ large compared to $d$. In particular, in the limit where $n$ goes to infinity, $\operatorname{Hilb}_{d}\left(\mathbf{A}^{\infty}\right)$ has the $\mathbf{A}^{1}$-homotopy type of the infinite Grassmannian $\operatorname{Gr}_{d-1}\left(\mathbf{A}^{\infty}\right) \simeq \mathrm{BGL}(d-1)$ [HJNTY20]. There are also corresponding stability theorems. In particular, over the complex numbers, the resulting homomorphism on integral cohomology,

$$
H^{*}(\operatorname{BGL}(d-1, \mathbf{C}), \mathbf{Z})=\mathbf{Z}\left[c_{1}, \ldots, c_{d-1}\right] \rightarrow H^{*}\left(\operatorname{Hilb}_{d}\left(\mathbf{A}^{n}\right), \mathbf{Z}\right),
$$

is an isomorphism in degrees at most $2 n-2 d+2$ [HJNTY20].

This paper considers another homotopical property of the Hilbert scheme Hilb ${ }_{d}\left(\mathbf{A}^{n}\right)$ for finite $n$. Namely, over the complex numbers, we show that $\operatorname{Hilb}_{d}\left(\mathbf{A}^{n}\right)$ (in the classical topology) has the homotopy type of Hilb $_{d}\left(\mathbf{A}^{n}, 0\right)$, the (compact) subspace of schemes supported at the origin (Corollary 2.3). This result is deduced from Theorem 2.2 on the topology of $\mathbf{G}_{m}$-actions. For example, it follows that the weight filtration on the rational cohomology $H^{i}\left(\mathrm{Hilb}_{d}\left(\mathbf{A}^{n}\right), \mathbf{Q}\right)$ is concentrated in weights $\leq i$, since that holds for proper schemes over C [Del75].

It remains open whether $\operatorname{Hilb}_{d}\left(\mathbf{A}^{n}, 0\right)$ is $\mathbf{A}^{1}$-homotopy equivalent to $\operatorname{Hilb}_{d}\left(\mathbf{A}^{n}\right)$, over the complex numbers or any other field. This would follow from our general Conjecture 2.1. We can say something about the unstable $\mathbf{A}^{1}$-homotopy type of these spaces, namely that $\operatorname{Hilb}_{d}\left(\mathbf{A}^{n}, 0\right)$ and $\operatorname{Hilb}_{d}\left(\mathbf{A}^{n}\right)$ are $\mathbf{A}^{1}$-connected (Theorems 7.1 and 7.3).

As a tool, we extend one of Bachmann's conservativity theorems, relating the motivic stable homotopy category to the derived category of motives along with real realizations (Theorem 8.1).

\section{Acknowledgments}

Thanks to Tom Bachmann, David Hemminger, Marc Hoyois, Joachim Jelisiejew, Denis Nardin, Maria Yakerson, and the referee for their suggestions.

\section{Main results, and a conjecture on $\mathbf{G}_{m}$-actions in motivic homotopy theory}

In this section, we formulate a general conjecture about actions of the multiplicative group $T=\mathbf{G}_{m}$ in motivic homotopy theory. (For motivic homotopy theory as defined by Morel and Voevodsky, a reference is [MV99] and an introduction is [AE17].) Roughly, if $T$ acts on a quasi-projective scheme $U$ which is attracted as $t \rightarrow 0$ in $T$ to a closed subset $Y$ in $U$, then the inclusion $Y \rightarrow U$ should be an $\mathbf{A}^{1}$-homotopy equivalence (Conjecture 2.1). We show that (over the complex numbers) the inclusion $Y \rightarrow U$ is at least a homotopy equivalence in the classical topology (Theorem 2.2, proved in Section 5).

Let $\mathrm{Hilb}_{d}\left(\mathbf{A}^{n}\right)$ be the quasi-projective scheme of zero-dimensional degree- $d$ closed subschemes of affine space $\mathbf{A}^{n}$ over a field $k$. When $k$ is the complex numbers, we deduce from Theorem 2.2 that $\operatorname{Hilb}_{d}\left(\mathbf{A}^{n}\right)$ has the homotopy type of $\operatorname{Hilb}_{d}\left(\mathbf{A}^{n}, 0\right)$, the (compact) subspace of schemes supported at the origin (Corollary 2.3).

Here is our general conjecture on actions of the multiplicative group. Let $X$ be a projective scheme over a field $k$ with an action of $T=\mathbf{G}_{m}$. Suppose that there is a $T$-equivariant ample line bundle on $X$. Let $Y$ be a $T$-invariant closed subset of $X$ such that every point $x$ in $X$ with $\lim _{t \rightarrow \infty}(t x) \in Y$ is in $Y$. Suppose that the fixed point set $Y^{T}$ is open in $X^{T}$. Let $U$ be the subset of points $x$ in $X$ such that $\lim _{t \rightarrow 0}(t x)$ is in $Y$. We show in Lemma 4.1 that $Y$ is contained in $U$ and $U$ is open in $X$. 
Conjecture 2.1. The inclusion $Y \rightarrow U$ is an $\mathbf{A}^{1}$-homotopy equivalence (that is, an isomorphism in the $\mathbf{A}^{1}$-homotopy category $\left.H(k)\right)$.

The assumption that $X$ has a $T$-equivariant ample line bundle is automatic if $X$ is normal [Sum75, Theorem 1.6].

Over the complex numbers, the proof of Theorem 2.2 shows that $Y$ is an attracting set for the $T$-action on $X$, in the terminology of topological dynamics, and $U$ is the basin of attraction for $Y$ [Mil85, section 1]. To say that $Y$ is an attracting set means that there is a neighborhood $N_{1}$ of $Y$ (in the classical topology) for which the images $t\left(N_{1}\right)$ converge to $Y$, meaning that for every neighborhood $N_{2}$ of $Y$, there is an $r>0$ such that $t\left(N_{1}\right) \subset N_{2}$ for all $t \in \mathbf{C}^{*}$ with $|t|<r$.

The conjecture would be useful for motivic homotopy theory, since $\mathbf{G}_{m}$-actions occur everywhere. When $U$ is smooth, both $Y$ and $U$ are unions of affine bundles over the connected components of $Y^{T}$, by Bialynicki-Birula [Bia73]. But even then, we only know how to prove that $Y \rightarrow U$ is an $\mathbf{A}^{1}$-homotopy equivalence after a suitable suspension (Theorem 9.1). Regardless of whether $U$ is smooth, the conjecture would be clear if the $T$-action on $U$ extended to a morphism

$$
\mathbf{A}^{1} \times U \rightarrow U,
$$

since $0 \times U$ would map into $Y$; but in general there is no such morphism. Even the $T$-action on $Y$ need not extend to a morphism $\mathbf{A}^{1} \times Y \rightarrow Y$ : consider the case where $Y$ is $\mathbf{P}^{1}$ with the standard action of $T$, where $\lim _{t \rightarrow 0} t x=0$ if $x \neq \infty$ but $\lim _{t \rightarrow 0} t(\infty)=\infty$.

Another way to describe the same situation is Drinfeld's analog of the Białynicki-Birula decomposition for singular varieties, although we will not use that explicitly in what follows. Namely, Drinfeld defines an algebraic space $Y^{+}$, the "attractor" of $Y$, as the space of $T$-equivariant morphisms $\mathbf{A}^{1} \rightarrow Y$; roughly speaking, a point of $Y^{+}$is a point $x$ of $Y$ together with a limit point $\lim _{t \rightarrow 0} t x$. Drinfeld shows that $Y^{+} \rightarrow Y$ is bijective for $Y$ proper over $k$, although usually not an isomorphism [Dril3, Proposition 1.4.11]. For example, if $Y=\mathbf{P}^{1}$ with the standard $T$-action, then the space $Y^{+}$is the disjoint union of $\mathbf{A}^{1}$ and the point at infinity. In a sense, the difficulty for Conjecture 2.1 is that the action of $T$ on $U$ does not extend to an action of the multiplicative monoid $\mathbf{A}^{1}$. The action of $T$ on $U^{+}$does extend to an action of $\mathbf{A}^{1}$; but that does not obviously help, because the morphism $U^{+} \rightarrow U$ is usually not a homotopy equivalence.

As evidence for Conjecture 2.1 in the singular case, we prove the following weaker statement in section 5. Theorem 2.2 was proved in the case where $U$ is smooth by Hausel and Rodriguez-Villegas [HR15, Corollary 1.3.6].

Theorem 2.2. Under the assumptions of Conjecture 2.1 with base field $\mathbf{C}$, the inclusion $Y \rightarrow U$ is a homotopy equivalence (in the classical topology).

Corollary 2.3. Over the complex numbers, the inclusion from $\operatorname{Hilb}_{d}\left(\mathbf{A}^{n}, 0\right)$ to $\mathrm{Hilb}_{d}\left(\mathbf{A}^{n}\right)$ (in the classical topology) is a homotopy equivalence.

Proof of Corollary 2.3. Let $X=\operatorname{Hilb}_{d}\left(\mathbf{P}^{n}\right)$ and $Y=\operatorname{Hilb}_{d}\left(\mathbf{A}^{n}, 0\right)$. The idea is to use the action of the multiplicative group $T$ (that is, $\mathbf{C}^{*}$ ) on $\operatorname{Hilb}_{d}\left(\mathbf{P}^{n}\right)$, coming from the action of $T$ on $\mathbf{P}^{n}$ by

$$
t\left(\left[x_{0}, \ldots, x_{n}\right]\right)=\left[x_{0}, t x_{1}, \ldots, t x_{n}\right]
$$

(We identify $\mathbf{A}^{n}$ with the open subset $x_{0} \neq 0$ in $\mathbf{P}^{n}$.) Here $X$ has a GL( $\left.n+1\right)$-equivariant ample line bundle by construction. (Namely, Grothendieck constructed the Hilbert scheme as a closed subscheme of the Grassmannian of subspaces of the vector space of homogeneous polynomials of sufficiently high degree, sending a closed subscheme $S \subset \mathbf{P}^{n}$ to the linear subspace of polynomials that vanish on $S$ [Kol95, Section I.1]. The standard ample line bundle $O(1)$ on the Grassmannian is $G L(n+1)$-equivariant.) In particular, $X$ has a $T$-equivariant ample line bundle. 
The open subset $U \subset X$ of 0 -dimensional schemes that converge as $t \in T$ approaches 0 to a subscheme supported at $[1,0, \ldots, 0]$ is exactly $\mathrm{Hilb}_{d}\left(\mathbf{A}^{n}\right)$. The action of $T$ on $U$ need not extend to a morphism $\mathbf{A}^{1} \times U \rightarrow U$ (or even $\mathbf{A}^{1} \times Y \rightarrow Y$ ). Nonetheless, the desired homotopy equivalence follows from Theorem 2.2 .

Remark 2.4. The action of $T$ on $Y=\operatorname{Hilb}_{d}\left(\mathbf{A}^{n}, 0\right)$ does not extend to a morphism $\mathbf{A}^{1} \times Y \rightarrow Y$ in any case where $Y \neq Y^{T}$. For example, for $Y=\operatorname{Hilb}_{3}\left(A^{2}, 0\right)$, the point $S_{a}:=\left\{x=a y^{2}, y^{3}=0\right\}$ in $Y$ has $\lim _{t \rightarrow 0} t\left(S_{a}\right)=Z:=\left\{x^{2}=0, x y=0, y^{2}=0\right\}$ for any $a \neq 0 \in \mathbf{C}$, whereas the point $S_{0}:=\left\{x=0, y^{3}=0\right\}$ in $Y$ is fixed by $T$ and hence has $\lim _{t \rightarrow 0} t\left(S_{0}\right)=S_{0}$.

\section{3. $\mathbf{G}_{m}$-actions and broken trajectories}

We show here that for an action of the multiplicative group $T=\mathbf{G}_{m}$ on a projective scheme, every limit of $T$-orbits is a broken trajectory, meaning a chain of $T$-orbits that connect a finite sequence of $T$-fixed points. This is analogous to fundamental results in Morse homology. Namely, given a smooth function on a closed Riemannian manifold satisfying some mild conditions, every limit of gradient flow lines is a broken trajectory, meaning a chain of gradient flow lines that connect a finite sequence of critical points [BH10, Theorem 4.9, Definition 4.10]. For a $T$-action on a smooth complex projective variety, one can in fact deduce the results here from those in Morse homology, applied to a Hamiltonian function for the $T$-action. Instead, we give a direct proof over any field. It turns out that smoothness is irrelevant.

Proposition 3.1. Let $X$ be a projective scheme over a field $k$ with an action of $T=\mathbf{G}_{m}$. Suppose that there is a $T$-equivariant ample line bundle on $X$. Then every limit of $T$-orbit closures in $X$ (in the Chow variety of effective 1-cycles on X) is a broken trajectory, that is, a chain of T-orbits (with some positive multiplicities) connecting some $T$-fixed points.

In more detail: let $C$ be a smooth curve over $k$ with a morphism $f: C \rightarrow X$, not mapping into $X^{T}$. Composing $f_{T}: T \times C \rightarrow T \times X$ with the action of $T$ gives a morphism $T \times C \rightarrow X$, which extends to a morphism $\mathbf{P}^{1} \times(C-Z) \rightarrow X$ for some O-dimensional closed subset $Z$ in $C$. This gives a morphism e from $C-Z$ to the Chow variety of 1-cycles on $X$, which extends to all of $C$ by properness of Chow varieties. Then for each $k$-point $c$ in $C$ (possibly in $Z$ ), $e(c)$ is a broken trajectory over $k$, meaning the sum of $T$-orbits (with some positive multiplicities) of points $y_{1}, \ldots, y_{n}$ in $X(k)$ that connect $T$-fixed points $x_{0}, \ldots, x_{n}$ in $X(k)$. More precisely, $\lim _{t \rightarrow 0} t\left(y_{i}\right)=x_{i-1}$ and $\lim _{t \rightarrow \infty} t\left(y_{i}\right)=x_{i}$ for each $1 \leq i \leq n$.

If the image of $f: C \rightarrow X$ is contained in $X^{T}$, then the morphism $e$ to the Chow variety of 1-cycles is constant (equal to zero as a 1-cycle). The proposition would still be true in that case if suitably interpreted: namely, any limit of $T$-fixed points in $X$ is a $T$-fixed point.

Proof. There is a $T$-equivariant embedding of $X$ into the projective space $P(V)$ for some representation $V$ of $T$. Given that, we can assume that $X=P(V)$; this greatly simplifies the situation. Then $T$ acts on $X=\mathbf{P}^{r}$ by $t\left(\left[z_{0}, \ldots, z_{r}\right]\right)=\left[t^{a_{0}} z_{0}, \ldots, t^{a_{r}} z_{r}\right]$ for some integers $a_{i}$. We can assume that $a_{0} \leq \cdots \leq a_{r}$.

Composing $f: C \rightarrow X$ with the action of $T$ on $X$ gives a morphism $T \times C \rightarrow X$, which can be viewed as a rational map $G: \mathbf{P}^{1} \times C \rightarrow X$ over $k$. Since $X$ is proper over $k, G$ becomes a morphism $W \rightarrow X$, where $W$ is a surface obtained by blowing up $\mathbf{P}^{1} \times C$ finitely many times at closed points. In particular, $G$ restricts to a morphism $\mathbf{P}^{1} \times(C-Z) \rightarrow X$ for some 0 -dimensional closed subset $Z$ of $X$.

Because $C$ is normal and all fibers of $W \rightarrow C$ have dimension 1, the fibers of $W \rightarrow C$ form a well-defined family of effective 1-cycles on $W$, and hence they give a morphism from $C$ to the Chow variety of 1-cycles on $W$ [Kol95, Theorem I.3.17]. Pushing cycles forward makes the Chow variety covariantly functorial under arbitrary morphisms [Kol95, Theorem I.6.8]. Therefore, the morphism $W \rightarrow X$ gives a morphism $e$ from $C$ to the Chow variety of 1-cycles on $X$. 


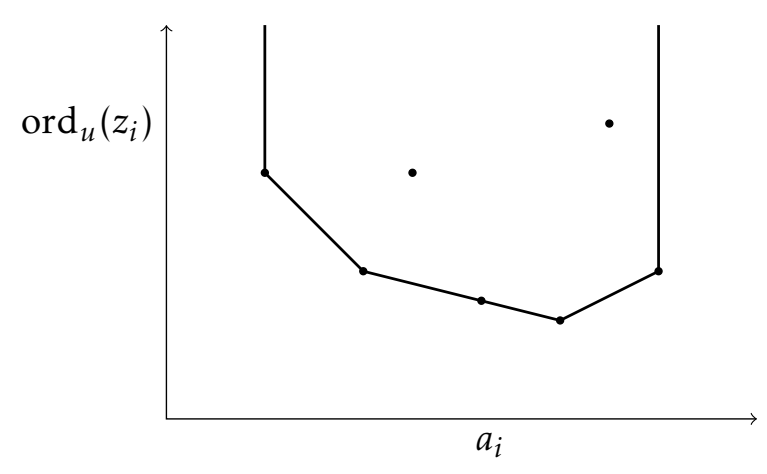

Figure 2. Newton polygon of the pairs $\left(a_{i}, \operatorname{ord}_{u}\left(z_{i}\right)\right)$

For each $k$-point $c$ in $C$ (possibly in $Z$ ), $e(c)$ is an effective 1-cycle in $X$ whose support $S$ is the image under $G$ of the inverse image of $c$ in $W$. Let us describe this image using power series. The completed local ring of $C$ at $c$ is isomorphic to the power series ring $k[[u]]$. So the curve $f: C \rightarrow X=\mathbf{P}^{r}$ near $c$ is given by some power series $\left[z_{0}(u), \ldots, z_{r}(u)\right]$ with $z_{i}(u) \in k[[u]]$, not all zero. Every point in the inverse image of $c$ in $W$ is contained in some curve in $W$ that meets the open set $T \times(C-Z)$ in $W$. After completion, this curve determines a finite extension $F$ of the field $k((u))$, along with an $F$-point of $T \times(C-Z)$ over the given $k((u))$-point of $C$. Therefore, the support $S$ of the limit 1-cycle $e(c)$, viewed as a subset of $X(\bar{k})$, is the set of all $\bar{k}$-points in $X$ that can be written as

$$
p=\lim _{u \rightarrow 0}\left[g(u)^{a_{0}} z_{0}(u), \ldots, g(u)^{a_{r}} z_{r}(u)\right]
$$

for some $g$ in the algebraic closure $\overline{k((u))}, g \neq 0$.

This limit point depends mainly on the rational number $b:=\operatorname{ord}_{u}(g)$. The situation is described by the Newton polygon of the pairs $\left(a_{i}, \operatorname{ord}_{u}\left(z_{i}\right)\right)$ in $\mathbf{Z} \times(\mathbf{Z} \cup \infty)$, as in Figure 2. (Here $\operatorname{ord}_{u}\left(z_{i}\right)=\infty$ if $z_{i}(u)$ is identically zero.)

Namely, let $I$ be the set of numbers $i \in\{0, \ldots, r\}$ such that $b a_{i}+\operatorname{ord}_{u}\left(z_{i}\right)$ reaches its minimum value. (That is, let $l$ be the unique line of slope $-b$ that meets the Newton polygon but not the region above it; then $I$ corresponds to the points $\left(a_{i}, \operatorname{ord}_{u}\left(z_{i}\right)\right)$ that lie on $l$.) Then we compute that the limit point $p$ defined above has all coordinates zero except the $i$ th coordinate for elements $i \in I$. Replacing $g$ by another function with the same value of $b$ (that is, multiplying $g$ by a unit $h(u)$ ) just replaces $p$ by $h(0)(p)$, another point in the same $T$-orbit as $p$.

For all but finitely many rational numbers $b$, the limit point $p$ above belongs to a set $\left\{x_{0}, x_{1}, \ldots, x_{n}\right\}$ of $T$-fixed points, these being indexed by the vertices of the Newton polygon. (For these values of $b$, all nonzero coordinates $i$ in the set $I$ above have the same weight $a_{i}$, which means that $p$ is a $T$-fixed point.) For the remaining $n$ values of $b$, corresponding to the non-vertical edges of the Newton polygon, the limit point can be anywhere in the $T$-orbit of a certain point $y_{i}$ in $X$ with $\lim _{t \rightarrow 0} t\left(y_{i}\right)=x_{i-1}$ and $\lim _{t \rightarrow \infty} t\left(y_{i}\right)=x_{i}$. Here the points $y_{1}, \ldots, y_{n}$ (and hence the points $x_{0}, x_{1}, \ldots, x_{n}$ ) can be taken to be $k$-points of $X$, by choosing the function $g \in \overline{k((u))}$ with a given value of $\operatorname{ord}_{u}(g) \in \mathbf{Q}$ to lie in a totally ramified extension of $k((u))$, for example in $k\left(\left(u^{1 / e}\right)\right)$ for a positive integer $e$.

\section{Openness}

We now prove that the subset $U$ attracted in $Y$ in Conjecture 2.1 is open in $X$.

Lemma 4.1. As in Conjecture 2.1, let $X$ be a projective scheme over a field $k$ with an action of $T=\mathbf{G}_{m}$. Suppose that there is a $T$-equivariant ample line bundle on $X$. Let $Y$ be a $T$-invariant closed subset of $X$ such that every 
point $x$ in $X$ with $\lim _{t \rightarrow \infty}(t x) \in Y$ is in $Y$. Suppose that the fixed point set $Y^{T}$ is open in $X^{T}$. Let $U$ be the subset of points $x$ in $X$ such that $\lim _{t \rightarrow 0}(t x)$ is in $Y$. Then $Y$ is contained in $U$, and $U$ is open in $X$.

Proof. Because $Y$ is a $T$-invariant closed subset of $X, Y$ is contained in $U$.

Clearly $U$ is a constructible subset of $X$; in Drinfeld's notation from section 2 above, $U$ is the image in $X$ of some connected components of $X^{+}$, those whose limit as $t \rightarrow 0$ is in $Y^{T}$. (By assumption, $Y^{T}$ is a union of some connected components of $X^{T}$.) It suffices to prove that $U$ is open in $X$ after replacing $k$ by its algebraic closure. If $U$ is not open in $X$, then there is a morphism $f$ from a smooth curve $C$ to $X$ with a $k$-point $c \in C$ such that $f(c) \in U$ and $f(d) \notin U$ for all $d \neq c$ in $C$. Thus $\lim _{t \rightarrow 0} t(f(d)) \in X^{T}-Y^{T}$ for $d \neq c$, whereas $\lim _{t \rightarrow 0} t(f(c)) \in Y^{T}$.

By Proposition 3.1, the limit of the T-orbit closures of the points $f(d)$ as $d$ approaches $c$ is a broken trajectory containing $f(c)$. By what we have said about $f(d)$, this broken trajectory ends (in the $t \rightarrow 0$ direction) at a point in $X^{T}-Y^{T}$. But this broken trajectory also contains $f(c)$ and hence the point $\lim _{t \rightarrow 0} t(f(c))$. Therefore, there is a broken trajectory from $\lim _{t \rightarrow 0} t(f(c)) \in Y^{T}$ down (in the $t \rightarrow 0$ direction) to a point in $X^{T}-Y^{T}$. This contradicts our assumption on $Y$, namely that $Y$ is a $T$-invariant closed subset such that every point $x$ in $X$ with $\lim _{t \rightarrow \infty}(t x) \in Y$ is in $Y$. We have shown that $U$ is open in $X$.

\section{Proof of Theorem 2.2}

Proof. To recall the assumptions: we have a projective scheme $X$ over $\mathbf{C}$ with an action of $T=\mathbf{C}^{*}$, and there is a $T$-equivariant ample line bundle on $X$. We have a $T$-invariant closed subscheme $Y$ of $X$ such that every point $x$ in $X$ with $\lim _{t \rightarrow \infty}(t x) \in Y$ is in $Y$, and the fixed point set $Y^{T}$ is open in $X^{T}$. Let $U$ be the subset of points $x$ in $X$ such that $\lim _{t \rightarrow 0}(t x)$ is in $Y$; then $U$ is Zariski open in $X$, and $Y$ is contained in $U$, by Lemma 4.1. We want to show that the inclusion $Y \rightarrow U$ is a homotopy equivalence in the classical topology.

Lemma 5.1. Let $q_{1}, q_{2}, \ldots$ be a sequence of complex points in $X$ that converge to a $T$-fixed point $w$. Let $t_{1}, t_{2}, \ldots$ be a sequence in $\mathbf{C}^{*}$ that converges to zero in $\mathbf{C}$. Then any limit point of the sequence $t_{i}\left(q_{i}\right)$ in $X$ lies in a broken trajectory "below $w$ ". That is, such a limit point belongs to the union of the T-orbits of some points $y_{1}, \ldots, y_{n}$ in $X$ and some $T$-fixed points $x_{0}, \ldots, x_{n}=w$ such that $\lim _{t \rightarrow 0} t\left(y_{i}\right)=x_{i-1}$ and $\lim _{t \rightarrow \infty} t\left(y_{i}\right)=x_{i}$ for each $1 \leq i \leq n$.

Proof. We largely follow the proof of Proposition 3.1. Choose a $T$-equivariant embedding of $X$ into $P(V)$ for some representation $V$ of $T$. We can write the action of $T$ on $\mathbf{P}^{r}=P(V)$ by $t\left(\left[z_{0}, \ldots, z_{r}\right]\right)=\left[t^{a_{0}} z_{0}, \ldots, t^{a_{r}} z_{r}\right]$ with $a_{0} \leq \cdots \leq a_{r}$. After passing to a subsequence, we can assume that the points $q_{1}, q_{2}, \ldots$ all have the same lowest weight $a_{j}$ of a nonzero coefficient. On the locally closed subset $K$ in $X$ of points with this lowest weight, the $T$-action $T \times K \rightarrow K$ extends to a morphism $f: \mathbf{A}^{1} \times K \rightarrow \bar{K}$, by inspection. Here $\bar{K}$ denotes the closure of $K$ in $X$.

By assumption, the points $\left(t_{i}, q_{i}\right)$ in $A_{1} \times K$ converge to the point $(0, w)$ in $\mathbf{A}^{1} \times \bar{K}$. The rational map $f: \mathbf{A}^{1} \times \bar{K} \rightarrow \bar{K}$ becomes a morphism after some blow-up $M \rightarrow \mathbf{A}^{1} \times \bar{K}$ that is an isomorphism over the complement of $0 \times(\bar{K}-K)$. So any limit point of the sequence $t_{i}\left(q_{i}\right)$ in $X$ is equal to $f(m)$ for some point $m$ in $M$ over $(0, w) \in \mathbf{A}^{1} \times \bar{K}$. In particular, we can choose a smooth algebraic curve with a morphism to $M$ that goes through $m$ and meets the open set $T \times K$.

Thus, by considering the completion of this curve at the point that maps to $m$, we have power series $g(u) \neq 0 \in \mathbf{C}[[u]]$ and $z(u) \in X(\mathbf{C}((u)))$ such that $g(0)=0, \lim _{u \rightarrow 0} z(u)=w$, and $\lim _{u \rightarrow 0}(g(u))(z(u))$ is the given limit point in $X$. The proof of Proposition 3.1 showed that the limit of the closures of $T$-orbits of $z(u)$ as $u$ approaches 0 is a broken trajectory in $X$, which clearly contains $w$ as one of the $T$-fixed points $x_{0}, \ldots, x_{n}$, say $w=x_{j}$. Moreover, since $g(0)=0$ (so that $\left.b:=\operatorname{ord}_{u}(g)>0\right)$, the explicit calculation of $\lim _{u \rightarrow 0}(g(u))(z(u))$ in $\mathbf{P}^{r}$ shows that this limit point is "below $w$ ", that is, in the union of $x_{0}, \ldots, x_{j}=w$ and the $T$-orbits that connect them. 
We continue the proof of Theorem 2.2. By the triangulation of real semialgebraic sets, there is a triangulation of $X$ with $Y$ as a subcomplex [Hir75, Section 1]. Therefore, $Y$ has arbitrarily small simplicial regular neighborhoods $N$ in $X$, and for these the inclusion $Y \rightarrow N$ is a homotopy equivalence [RS72, Chapter 3]. Let $N$ be a (compact) regular neighborhood of $Y$ contained in $U$.

Consider the submonoid $(0,1]$ of $T=\mathbf{C}^{*}$. It would be convenient to have $(0,1] \cdot N \subset N$, but it is not obvious that we can arrange that. Instead, we argue as follows. I claim that each point $w \in Y^{T}$ has a neighborhood $N_{1}$ in $U$ such that $t\left(N_{1}\right) \subset N$ for all $t \in(0,1]$. If not, then there would be a sequence $q_{i}$ in $U$ converging to $w$ such that for each positive integer $j,(0,1] \cdot q_{j}$ is not contained in $N$. So there is a sequence $t_{i} \in(0,1]$ such that $t_{i}\left(q_{i}\right)$ is not in $N$. The sequence $t_{i}$ must converge to zero; otherwise, a subsequence of $t_{i}\left(q_{i}\right)$ would converge to the $T$-fixed point $w$ in $Y$ (and hence infinitely many of those points would be in $N$ ).

After passing to subsequences, we can assume that $t_{i}\left(q_{i}\right)$ converges to a point $v$ in $X-\operatorname{int}(N)$, hence not in $Y$. By Lemma 5.1, $v$ belongs to the union of some finite chain of $T$-orbits going "down" from $w$, meaning the $T$-orbits of some points $y_{1}, \ldots, y_{n}$ in $X$ and some $T$-fixed points $x_{0}, \ldots, x_{n}=w$ such that $\lim _{t \rightarrow 0} t\left(y_{i}\right)=x_{i-1}$ and $\lim _{t \rightarrow \infty} t\left(y_{i}\right)=x_{i}$. By our assumption that all points $x$ in $X$ with $\lim _{t \rightarrow \infty} t x \in Y$ are in $Y$, it follows that $v$ is in $Y$, a contradiction. Thus we have proved the claim that each point $w \in Y^{T}$ has a neighborhood $N_{1}$ in $U$ such that $t\left(N_{1}\right) \subset N$ for all $t \in(0,1]$.

More generally, for each point $x \in U$ (not just in $Y^{T}$ ), there is a real number $a \in(0,1]$ and a neighborhood $N_{1}$ of $x$ in $U$ such that $t\left(N_{1}\right) \subset N$ for all $t \in(0, a]$. That follows from the previous statement applied to the point $y=\lim _{t \rightarrow 0} t(x) \in Y^{T}$.

Therefore, for every compact subset $K$ of $U$, there is a real number $a \in(0,1]$ such that $t(K) \subset N$ for all $t \in(0, a]$. Equivalently, $K \subset a^{-1}(N)$. In particular, there is a real number $c>1$ such that the compact neighborhood $N$ of $Y$ is contained in the interior of $c(N)$. It also follows that $U$ is the union of the subsets $c^{j}(N)$ over all $j \geq 0$.

Since the inclusion $Y \rightarrow N$ is a homotopy equivalence, so is the inclusion $Y \rightarrow c^{j}(N)$ for each integer $j$. Therefore, each of the inclusions $c^{j}(N) \rightarrow c^{j+1}(N)$ is also a homotopy equivalence. Since $c^{j}(N)$ is a closed subset contained in the interior of $c^{j+1}(N)$, the union of these subsets (namely, $U$ ) has the colimit topology. Since this is a filtered colimit, the colimit $U$ is equivalent to the homotopy colimit, and so the inclusion $N \rightarrow U$ is a homotopy equivalence. Since the inclusion $Y \rightarrow N$ is also a homotopy equivalence, we conclude that $Y \rightarrow U$ is a homotopy equivalence.

\section{The real case}

Theorem 6.1. Under the assumptions of Conjecture 2.1 with base field $\mathbf{R}$, the inclusion $Y(\mathbf{R}) \rightarrow U(\mathbf{R})$ is a homotopy equivalence.

Proof. This is similar to the complex case (Theorem 2.2). In particular, Lemma 5.1 holds by the same proof over $\mathbf{R}$ in place of $\mathbf{C}$, using that Proposition 3.1 expresses any limit of $T$-orbits of $\mathbf{R}$-points as the union of a finite chain of $T$-orbits of $\mathbf{R}$-points. Given that, the proof of Theorem 2.2 applies verbatim (using a regular neighborhood of $Y(\mathbf{R})$ inside $U(\mathbf{R}))$ to show that the inclusion $Y(\mathbf{R}) \rightarrow U(\mathbf{R})$ is a homotopy equivalence.

\section{7. $\mathrm{A}^{1}$-connectedness of the Hilbert scheme}

Hartshorne showed that the Hilbert scheme of projective space over a field $k$ (of subschemes with a given Hilbert polynomial) is connected [Har66]. In particular, $\operatorname{Hilb}_{d}\left(\mathbf{P}^{n}\right)$ is connected for every $n \geq 1$ and $d \geq 0$. The argument was sharpened by Reeves and Pardue [Ree95, Par96]. Reeves and Pardue showed that for an infinite field $k$, any two $k$-points of $\mathrm{Hilb}_{d}\left(\mathbf{P}^{n}\right)$ can be connected by a chain of affine lines over $k$. By Morel's results (Lemma 7.2 below), it follows that $\operatorname{Hilb}_{d}\left(\mathbf{P}_{k}^{n}\right)$ is $\mathbf{A}^{1}$-connected for $k$ infinite. 
We now show that $\operatorname{Hilb}_{d}\left(\mathbf{A}^{n}\right)$ and $\operatorname{Hilb}_{d}\left(\mathbf{A}^{n}, 0\right)$ are $\mathbf{A}^{1}$-connected over an infinite field $k$. This seems to be harder for $\mathrm{Hilb}_{d}\left(\mathbf{A}^{n}, 0\right)$, because (for $d>1$ ) this space contains no smooth subschemes of $\mathbf{A}^{n}$. When $n \geq d$, the $\mathbf{A}^{1}$-connectedness of these Hilbert schemes can be proved using the ideas of [HJNTY20], but here we want the results for all $n$ and $d$.

Theorem 7.1. Let $k$ be an infinite field, $n \geq 1, d \geq 0$. Then $\operatorname{Hilb}_{d}\left(\mathbf{A}^{n}\right)$ is $\mathbf{A}^{1}$-connected over $k$.

Proof. We use the following result of Morel's:

Lemma 7.2. Let $X$ be a separated scheme of finite type over a field $k$ such that $X$ has a $k$-point. Suppose that for every separable finitely generated field extension $F$ of $k$, any two $F$-points of $X$ can be connected by a chain of affine lines $\mathbf{A}_{F}^{1} \rightarrow X_{F}$. Then $X$ is $\mathbf{A}^{1}$-connected.

Proof. For $m \geq 0$, Morel showed that an $\mathbf{A}^{1}$-local pointed simplicial Nisnevich sheaf $X$ over $k$ is $m$-connected if and only if the fiber $X(F)$ is $m$-connected for every separable finitely generated field extension $F$ of $k$ [Mor05, Lemma 6.1.3]. Also, for a simplicial sheaf $X, \pi_{0}(X) \rightarrow \pi_{0}^{\mathbf{A}^{1}}(X)$ is a surjection of Nisnevich sheaves [MV99, Section 2, Corollary 3.22]. In particular, for a separated scheme $X$ of finite type over $k$, $X(F) \rightarrow \pi_{0}^{\mathbf{A}^{1}}(X)(F)$ is surjective for every separable finitely generated field extension $F$ over $k$. This implies the lemma.

By Lemma 7.2, it suffices to show that for an infinite field $k$, any two $k$-points of $U:=\operatorname{Hilb}_{d}\left(\mathbf{A}^{n}\right)$ can be connected by a chain of affine lines $\mathbf{A}_{k}^{1} \rightarrow U_{k}$. So let $S$ be any $k$-point of $U$. That is, $S$ is a closed subscheme of $\mathbf{A}^{n}$ over $k$ of dimension zero and degree $d$. We use a "Gröbner degeneration", as follows. Let $c$ be a large positive integer, and consider the action of $T:=\mathbf{G}_{m}$ on $\mathbf{A}^{n}$ by

$$
t\left(x_{1}, \ldots, x_{n}\right)=\left(t^{c} x_{1}, t^{c^{2}} x_{2}, \ldots, t^{c^{n}} x_{n}\right) .
$$

Then $S_{2}:=\lim _{t \rightarrow 0} t(S)$ exists in $U$. It is a closed subscheme supported at the origin in $\mathbf{A}^{n}$, and it is fixed by this $T$-action. That is, the defining ideal $I$ of $S_{2}$ as a subscheme of $\mathbf{A}_{k}^{n}$ is homogeneous with respect to the weights $\left(c, c^{2}, \ldots, c^{n}\right)$ on $x_{1}, \ldots, x_{n}$. Taking $c$ big enough compared to $d$ and $n$, it follows that $I$ is generated by monomials. By construction, we can connect $S$ to $S_{2}$ by an affine line over $k$.

Since $S_{2}$ has dimension 0 and is defined by monomials, it is smoothable, using Hartshorne's proof by distraction; a specific reference is [CEVV09, Proposition 4.15]. We need the more precise information given by the proof, as follows. Let $I=\left(x^{M_{1}}, \ldots, x^{M_{r}}\right)$ be the minimal set of monomial generators for the ideal $I$. We use multi-index notation, so $x^{M_{i}}=\prod_{j=1}^{n} x_{j}^{M_{i j}}$. Consider the following flat family of ideals in $k\left[x_{1}, \ldots, x_{n}\right]$ parametrized by affine space $A^{d}$ : for a point $\left(a_{0}, \ldots, a_{d-1}\right)$ in $A^{d}$, take the ideal $J_{a}$ in $k\left[x_{1}, \ldots, x_{n}\right]$ generated by the elements

$$
f_{i}:=\prod_{j=1}^{n}\left(x_{j}-a_{0}\right)\left(x_{j}-a_{1}\right) \cdots\left(x_{j}-a_{M_{i j}-1}\right) .
$$

The initial ideal of $J_{a}$ (with respect to any monomial order compatible with the grading, say the graded reverse lexicographic order) is $I$; so we have a flat family. This defines a morphism $A^{d} \rightarrow \operatorname{Hilb}_{d}\left(\mathbf{A}^{n}\right)$ over $k$, with the origin mapping to the given monomial scheme $S_{2}$. When $a_{0}, \ldots, a_{d-1}$ are distinct elements of $k$, the subscheme $Z_{a}$ of $\mathbf{A}^{n}$ defined by $J_{a}$ contains $d$ distinct $k$-points: namely, for each of the $d$ monomials $x^{L}$ not in $I, Z_{a}$ contains the $k$-point $\left(a_{L_{1}}, \ldots, a_{L_{n}}\right)$. Since the scheme $Z_{a}$ has degree $d$, it must be smooth over $k$, equal to those $d k$-points in $\mathbf{A}^{n}$.

Since $k$ is infinite, it follows that we can connect $S_{2}$ by an affine line in $\operatorname{Hilb}_{d}\left(\mathbf{A}^{n}\right)$ to a scheme $S_{3}$ which consists of $d$ distinct $k$-points in $\mathbf{A}^{n}$. If $n \geq 2$, since the condition for two points to be equal in $\mathbf{A}^{n}$ has codimension at least 2 , it is easy to connect $S_{3}$ by a chain of affine lines over $k$ to a fixed arrangement $S_{4}$ of $d$ distinct $k$-points in $\mathbf{A}^{n}$. Thus Hilb ${ }_{d}\left(\mathbf{A}^{n}\right)$ is $\mathbf{A}^{1}$-connected when $n \geq 2$. It is also $\mathbf{A}^{1}$-connected when $n=1$, since $\operatorname{Hilb}_{d}\left(\mathbf{A}^{1}\right) \cong A^{d}$. 
Theorem 7.3. Let $k$ be an infinite field, $n \geq 1, d \geq 0$. Then $\operatorname{Hilb}_{d}\left(\mathbf{A}^{n}, 0\right)$ is $\mathbf{A}^{1}$-connected over $k$.

Proof. By Lemma 7.2, it suffices to show that for every infinite field $k$, any two $k$-points of $Y:=\operatorname{Hilb}_{d}\left(\mathbf{A}^{n}, 0\right)$ can be connected by a chain of affine lines over $k$. For lack of a direct proof, we will reduce this to Theorem 7.1.

Let $X=\operatorname{Hilb}_{d}\left(\mathbf{P}^{n}\right), U=\operatorname{Hilb}_{d}\left(\mathbf{A}^{n}\right)$, and $T=\mathbf{G}_{m}$. Consider the action of $T$ on $X$ coming from the action of $T$ by scaling on $\mathbf{A}^{n}$. Then $Y$ is a $T$-invariant closed subset of $U$, and $\lim _{t \rightarrow 0} t(x)$ exists in $Y$ for each point $x$ in $U$. Clearly we can connect any $k$-point $x$ in $Y$ to this limit point by an affine line in $Y$, and the limit point is fixed by $T$. So it suffices to show that any two $k$-points $p, q$ in $Y^{T}$ can be connected by a chain of affine lines in $Y$.

We know by the proof of Theorem 7.1 that $p$ and $q$ can be connected by a chain of affine lines in $U$. So it suffices to show that for any morphism $f: \mathbf{A}^{1} \rightarrow U$ over $k$, we can connect $\lim _{t \rightarrow 0} t(f(0))$ to $\lim _{t \rightarrow 0} t(f(1))$ by a chain of affine lines in $Y$.

Composing $f$ with the action of $T$ on $U$ gives a morphism $T \times \mathbf{A}^{1} \rightarrow U$ over $k$, which can be viewed as a rational map $\mathbf{P}^{1} \times \mathbf{P}^{1} \rightarrow X$ over $k$. Since $X$ is proper over $k$, this map becomes a morphism after blowing up the domain finitely many times at closed points. It follows that $g(s):=\lim _{t \rightarrow 0} t(f(s)) \operatorname{defines}$ a morphism $g: \mathbf{A}^{1}-Z \rightarrow Y$ for some 0 -dimensional closed subset $Z$ of $\mathbf{A}^{1}$. Since $Y$ is proper over $k, g$ extends to a morphism $g: \mathbf{A}^{1} \rightarrow Y$. As a result, for any two $k$-points $s_{1}, s_{2}$ in $\mathbf{A}^{1}-Z, \lim _{t \rightarrow 0} t\left(f\left(s_{1}\right)\right)$ and $\lim _{t \rightarrow 0} t\left(f\left(s_{2}\right)\right)$ can be connected by an affine line in $Y$.

There remains the case where 0 or 1 is in $Z$. It suffices to show that for any $k$-point $s_{0}$ in $Z$ (which will be 0 or 1 for us), the point $z_{0}:=\lim _{t \rightarrow 0} t\left(f\left(s_{0}\right)\right)$ can be connected by a chain of affine lines in $Y$ to $g\left(s_{0}\right)$.

By Proposition 3.1, the T-orbits of the points $f(s)$ (for $s \in \mathbf{A}^{1}-Z$ ) converge as $s$ approaches $s_{0}$ to a "broken trajectory" containing $f\left(s_{0}\right)$. This means the union of $T$-orbits of points $y_{1}, \ldots, y_{n}$ in $X(k)$ that connect $T$-fixed points $x_{0}, \ldots, x_{n}$ in $X(k)$, in the sense that $\lim _{t \rightarrow 0} t\left(y_{i}\right)=x_{i-1}$ and $\lim _{t \rightarrow \infty} t\left(y_{i}\right)=x_{i}$.

Both the $k$-point $z_{0}=\lim _{t \rightarrow 0} t\left(f\left(s_{0}\right)\right)$ and the $k$-point $g\left(s_{0}\right)$ lie in this union of $T$-orbit closures in $X$, and both are in the closed subset $Y$. We know that every point $x$ in $X$ with $\lim _{t \rightarrow \infty}(t x) \in Y$ is in $Y$. Therefore, all the orbit closures that connect $z_{0}$ to $g\left(s_{0}\right)$ are in $Y$. So these two points can be connected by affine lines over $k$ in $Y$, as we want.

\section{Conservativity for the motivic stable homotopy category}

Extending one of Tom Bachmann's results, we prove the following conservativity theorem, relating the motivic stable homotopy category with the derived category of motives along with real realizations. Thanks for Bachmann for his suggestions. This result will be used in the proof of Theorem 9.1.

Theorem 8.1. Let $k$ be a finitely generated field of characteristic zero (that is, a finitely generated extension field of $\mathbf{Q}$. Let $A$ be a compact object in $S H(k)$ such that $M(A)=0$ in $D M(k)$ and for every embedding of $k$ into $\mathbf{R}$, $H_{*}(A(\mathbf{R}), \mathbf{Z}[1 / 2])=0$. Then $A=0$.

If $k$ has no real embedding, Theorem 8.1 just says that $M(A)=0$ in $D M(k)$ implies $A=0$ in $S H(k)$.

Proof. Bachmann showed (in particular) that if $A$ is a compact object in $S H(k)$ such that $M(A)=0$ in $D M(k)$ and for every $\sigma$ in the space $\operatorname{Sper}(k)$ of orderings of $k, M_{\sigma}[1 / 2](A)=0$ in $D(\mathbf{Z}[1 / 2])$, then $A=0[\operatorname{Bac} 18$, Theorem 33]. When $\sigma$ comes from an embedding of $k$ into $\mathbf{R}, M_{\sigma}[1 / 2](A)$ is the complex that computes the singular homology of the corresponding real realization of $A, H_{*}(A(\mathbf{R}), \mathbf{Z}[1 / 2])$ [Bac18, Remark 1]. It remains to show that we only need to consider orderings that come from real embeddings of $k$.

We use the following property of the space $\operatorname{Sper}(k)$ of orderings of $k$ [FHV94, Lemma 1.6]. The topology on $\operatorname{Sper}(k)$ is defined by taking the sets $\left\{\sigma: a>_{\sigma} 0\right\}$ for $a \in k$ as a sub-basis for the topology. This makes $\operatorname{Sper}(k)$ into a compact Hausdorff totally disconnected space. 
Lemma 8.2. Let $k$ be a finitely generated field of characteristic zero. Then the set of archimedean orderings of $k$ is dense in the topological space $\operatorname{Sper}(k)$ of orderings of $k$, and every archimedean ordering comes from an embedding of $k$ into $\mathbf{R}$.

Given that, we are done if we can show that the support in $X:=\operatorname{Sper}(k)$ of a compact object in $S H(k)$ is open as well as closed. This is related to the general fact that for a tensor triangulated category $K$, the support of an object of $K$ in the Balmer spectrum $\operatorname{Spc}(K)$ is closed and its complement is quasi-compact [Bal05, Proposition 2.14]. However, we will argue more directly.

We use that the functors $M_{\sigma}$ come from a functor from $S H(k)$ to the derived category of sheaves $D(X, \mathbf{Z}[1 / 2])$, which takes compact objects to compact objects. Indeed, by [Bac18, Lemma 21], the functor from $S H(k)$ to Witt motives $D M_{W}(k, \mathbf{Z}[1 / 2])$ is monoidal; so it takes rigid objects to rigid objects, and the rigid objects coincide with the compact objects in these categories. (Some people say "strongly dualizable" rather than "rigid".) Furthermore, $D M_{W}(k, \mathbf{Z}[1 / 2])$ is equivalent to $D(X, \mathbf{Z}[1 / 2])$ [Bac18, Lemma 26 and proof of Theorem 30].

A compact object in $D(X, Z[1 / 2])$ is a perfect complex; that is, it is locally isomorphic to a bounded complex of finitely generated projective $\mathbf{Z}[1 / 2]$-modules. (Indeed, since $X$ is compact, Hausdorff, and totally disconnected, every open subset of $X$ is a union of clopen subsets (or equivalently, quasi-compact open subsets). It follows that every compact object in $D(X, \mathbf{Z}[1 / 2])$ is a summand in $D(X, \mathbf{Z}[1 / 2])$ of a bounded complex of sheaves which are finite direct sums of sheaves of the form $j_{!}\left(\mathbf{Z}[1 / 2]_{U}\right)$, with $j: U \hookrightarrow X$ the inclusion of a quasi-compact open subset [Stacks, Lemma 094C]. Clearly such a summand is a perfect complex of $\mathbf{Z}[1 / 2]$-modules on $X$.)

Because sections of the sheaf $\mathbf{Z}[1 / 2]$ on $X=\operatorname{Sper}(k)$ are locally constant, the support of a perfect complex on $X$ is open as well as closed.

\section{9. $\mathbf{G}_{m}$-actions on smooth varieties and motivic homotopy theory}

We now consider Conjecture 2.1 in the special case where $U$ is smooth. (One example where this applies is the inclusion from $\operatorname{Hilb}_{d}\left(A^{2}, 0\right)$ to $\mathrm{Hilb}_{d}\left(A^{2}\right)$.) For $U$ smooth, we show that the inclusion $Y \rightarrow U$ becomes an $\mathbf{A}^{1}$-homotopy equivalence after suspending by $\mathbf{S}^{3,1}=\mathbf{S}^{2} \wedge \mathbf{G}_{m}$. It follows that $Y$ and $U$ have many invariants in common, such as motivic homology and cohomology, $l$-adic cohomology, and so on. On the other hand, it remains open whether the Nisnevich sheaf $\pi_{0}^{\mathbf{A}^{1}}$ is the same for $Y$ and $U$, and likewise for $\pi_{1}^{\mathbf{A}^{1}}$. At least for $\pi_{0}^{\mathbf{A}^{1}}$, one might hope to imitate the proof of Theorem 7.3.

Theorem 9.1. Under the assumptions of Conjecture 2.1 with base field $k$ of characteristic zero, and assuming that $U$ is smooth over $k$, the inclusion $Y \rightarrow U$ becomes an $\mathbf{A}^{1}$-homotopy equivalence after suspending by $\mathbf{S}^{3,1}=\mathbf{S}^{2} \wedge \mathbf{G}_{m}$.

Proof. We can assume that $U$ is connected, by arguing separately for each connected component of $U$. Next, by equivariant resolution of singularities (using that $k$ has characteristic zero), we can assume that $X$ (as well as $U$ ) is smooth over $k$, while still having a $T$-action [Kol07, Proposition 3.9.1].

We first show that the inclusion $Y \rightarrow U$ induces an isomorphism in the derived category of motives $D M(k), M(Y) \rightarrow M(U)$. Namely, since $X$ is smooth over $k$, we have the Bialynicki-Birula decomposition, as follows. The fixed point set $X^{T}$ is smooth over $k$. Write $Z_{1}, \ldots, Z_{m}$ for the connected components of $X^{T}$. For each $i$, let $Z_{i}^{+}=\left\{x \in X: \lim _{t \rightarrow 0} t x \in Z_{i}\right\}$ and $Z_{i}^{-}=\left\{x \in X: \lim _{t \rightarrow \infty} t x \in Z_{i}\right\}$ be the stable and unstable manifolds of $Z_{i}$. Then the action of $T$ gives morphisms $Z_{i}^{+} \rightarrow Z_{i}$ and $Z_{i}^{-} \rightarrow Z_{i}$ which are affine-space bundles [Bia73].

Karpenko showed that this geometric decomposition gives a direct-sum decomposition of Chow motives over $k$ [Kar00, Theorem 6.5], [Bro05, Theorem 3.5]:

$$
M(X) \cong \oplus_{i=1}^{m} M\left(Z_{i}\right)\left\{a_{i}\right\},
$$


where $a_{i}:=\operatorname{dim}\left(Z_{i}^{+}\right)-\operatorname{dim}\left(Z_{i}\right)$. (Here $\mathbf{Z}\{1\}$ denotes the Lefschetz motive, with $M\left(\mathbf{P}^{1}\right)=\mathbf{Z}\{0\} \oplus \mathbf{Z}\{1\}$.) This implies another decomposition $M(X) \cong \oplus_{i=1}^{m} M\left(Z_{i}\right)\left\{b_{i}\right\}$, where $b_{i}:=\operatorname{dim}\left(Z_{i}^{-}\right)-\operatorname{dim}\left(Z_{i}\right)$, by inverting the $T$-action on $X$. Here

$$
a_{i}+\operatorname{dim}\left(Z_{i}\right)+b_{i}=n,
$$

by considering the action on $T$ on the tangent space to $X$ at a point of $Z_{i}$.

The category of Chow motives is a full subcategory of the derived category of motives, $D M(k)$ : the thick subcategory generated by smooth projective schemes over $k$ tensored with $\mathbf{Z}\{a\}=\mathbf{Z}(a)[2 a]$ for integers $a$ [Voe00]. Every scheme $X$ of finite type over $k$ has a motive $M(X)$ and a compactly supported motive $M^{c}(X)$ in $D M(k)$. We can assume that $Z_{1}, \ldots, Z_{m}$ are ordered in such a way that the closure of $Z_{i}^{-}$is contained in $X_{i}:=\cup_{j \leq i} Z_{j}^{-}$. Karpenko's argument shows that the exact triangle

$$
M^{c}\left(X_{i-1}\right) \rightarrow M^{c}\left(X_{i}\right) \rightarrow M^{c}\left(Z_{i}^{-}\right) \cong M\left(Z_{i}\right)\left\{b_{i}\right\}
$$

in $D M(k)$ is split [Kar00, Theorem 6.5, part (a)]. (Indeed, his splitting on Chow groups is defined by an element of $C H_{\operatorname{dim}\left(Z_{i}\right)+b_{i}}\left(Z_{i} \times X_{i}\right)$, and that is precisely $\operatorname{Hom}\left(M\left(Z_{i}\right)\left\{b_{i}\right\}, M^{c}\left(X_{i}\right)\right)$ since $Z_{i}$ is smooth and proper over $k$.) In particular, it follows that

$$
M\left(X_{j}\right) \cong \oplus_{i=1}^{j} M\left(Z_{i}\right)\left\{b_{i}\right\}
$$

for each $1 \leq j \leq m$, and its open complement $X-X_{j}$ satisfies

$$
M^{c}\left(X-X_{j}\right) \cong \oplus_{i=j+1}^{m} M\left(Z_{i}\right)\left\{b_{i}\right\}
$$

(Here $X_{j}$ need not be smooth, but it is proper over $k$, and so its motive $M\left(X_{j}\right)$ is the same as its compactly supported motive $M^{c}\left(X_{j}\right)$.)

In the notation of Conjecture 2.1, we can assume that the closed subset $Y$ of $X$ is equal to $X_{r}$ for some $r \leq m$. So $M(Y) \cong \oplus_{i=1}^{r} M\left(Z_{i}\right)\left\{b_{i}\right\}$. Likewise, the open subset $U$ is the union of the subsets $Z_{i}^{+}$with $i \leq r$. By the splitting in $D M(k)$ above, applied to the inverse action of $T$ on $X$, we have

$$
M^{c}(U) \cong \oplus_{i=1}^{r} M\left(Z_{i}\right)\left\{a_{i}\right\} .
$$

Since $U$ is smooth of dimension $n$ over $k$, it follows that

$$
\begin{aligned}
M(U) & \cong M^{c}(U)^{*}\{n\} \\
& \cong \oplus_{i=1}^{r} M\left(Z_{i}\right)^{*}\left\{n-a_{i}\right\} \\
& \cong \oplus_{i=1}^{r} M\left(Z_{i}\right)\left\{n-a_{i}-\operatorname{dim}\left(Z_{i}\right)\right\} \\
& \cong \oplus_{i=1}^{r} M\left(Z_{i}\right)\left\{b_{i}\right\} .
\end{aligned}
$$

Thus $M(Y)$ is isomorphic to $M(U)$ in $D M(k)$. More precisely, the inclusion $Y \rightarrow U$ induces an isomorphism $M(Y) \rightarrow M(U)$. To see this, one checks from Karpenko's construction of the splittings that for $i, j \in\{1, \ldots, r\}$, the composition $M\left(Z_{i}\right)\left\{b_{i}\right\} \rightarrow M(Y) \rightarrow M(U) \rightarrow M\left(Z_{j}\right)\left\{b_{j}\right\}$ is the identity for $i=j$ and zero if $i<j$.

The schemes $Y, U, X$ with $T$-action are defined over some finitely generated subfield of $k$. So we can assume that the field $k$ is finitely generated over $\mathbf{Q}$. Apply Theorem 8.1 to the cofiber $A=\Sigma^{\infty}(U / Y)$ in $S H(k)$. We showed above that the motive of $A$ in $D M(k)$ is zero. Also, for every real embedding of $k$, the real realization of $A$ is zero in the stable homotopy category, by Theorem 6.1. (It may be that $k$ has no real embedding.) Therefore, $A=0$ in $S H(k)$. That is, the inclusion $Y \rightarrow U$ induces an isomorphism in $S H(k)$.

Again using that $k$ has characteristic zero, Bachmann showed that the $\mathbf{P}^{1}$-suspension functor

$$
Q=\Sigma_{\mathbf{P}^{1}}^{\infty}: H(k)_{*} \rightarrow S H(k)
$$

is conservative on $\mathbf{A}^{1}$-simply connected spaces which can be written as homotopy colimits of spaces $X_{+} \wedge \mathbf{G}_{m}$ with $X \in \mathrm{Sm}_{k}$ [Bac21, Theorem 1.3]. 
The $\mathbf{S}^{2}$-suspension of every space in $H(k)_{*}$ is $\mathbf{A}^{1}$-simply connected. The map $\mathbf{S}^{2} \wedge \mathbf{G}_{m} \wedge Y_{+} \rightarrow \mathbf{S}^{2} \wedge \mathbf{G}_{m} \wedge U_{+}$ is therefore a pointed $\mathbf{A}^{1}$-homotopy equivalence.

Theorem 9.1 can be slightly strengthened if in addition $Y$ and $U$ are $\mathbf{A}^{1}$-connected. In that case, their $\mathbf{S}^{1}$-suspensions are $\mathbf{A}^{1}$-simply connected, and so $\mathbf{P}^{1} \wedge Y_{+} \rightarrow \mathbf{P}^{1} \wedge U_{+}$is a pointed $\mathbf{A}^{1}$-homotopy equivalence, using that $\mathbf{P}^{1}=\mathbf{S}^{2,1}=\mathbf{S}^{1} \wedge \mathbf{G}_{m}$.

\section{References}

[AE17] B. Antieau and E. Elmanto, A primer for unstable motivic homotopy theory, in: Surveys on recent developments in algebraic geometry, Proc. Sympos. Pure Math. 95, 305-370. Amer. Math. Soc. (2017).

[Bac18] T. Bachmann, On the conservativity of the functor assigning to a motivic spectrum its motive, Duke Math. J. 167 (2018), 1525-1571.

[Bac21] _ The zeroth $\mathbf{P}^{1}$-stable homotopy sheaf of a motivic space, J. Inst. Math. Jussieu (2021), 1-25.

[Bal05] P. Balmer, The spectrum of prime ideals in tensor triangulated categories, J. Reine Angew. Math. 588 (2005), 149-168.

[BH10] A. Banyaga and D. Hurtubise, Morse-Bott homology, Trans. Amer. Math. Soc. 362 (2010), 39974043.

[Bia73] A. Białynicki-Birula, Some theorems on actions of algebraic groups, Annals of Math. 98 (1973), 480-497.

[Bro05] P. Brosnan, On motivic decompositions arising from the method of Bialynicki-Birula, Invent. Math. 161 (2005), 91-111.

[CEVV09] D. Cartwright, D. Erman, M. Velasco, and B. Viray, Hilbert schemes of 8 points, Algebra Number Theory 3 (2009), 763-795.

[Del75] P. Deligne, Poids dans la cohomologie des variétés algébriques, in: Proceedings of the International Congress of Mathematicians (Vancouver, 1974), 79-85. Canad. Math. Congress, Montreal (1975).

[Dri13] V. Drinfeld, On algebraic spaces with an action of $\mathbf{G}_{m}$, preprint arXiv:1308.2604 (2013).

[FHV94] M. Fried, D. Haran, and H. Völklein. Real Hilbertianity and the field of totally real numbers, in: Arithmetic geometry. Conference on arithmetic geometry with an emphasis on Iwasawa theory, (Arizona State University, 1993), Contemp. Math. 174, 1-34 (1994).

[Göt02] L. Göttsche, Hilbert schemes of points on surfaces, in: Proceedings of the International Congress of Mathematicians (Beijing, 2002), Vol. II, 483-494. Higher Ed. Press, Beijing (2002).

[Har66] R. Hartshorne, Connectedness of the Hilbert scheme, Inst. Hautes Étud. Sci. Publ. Math. 29 (1966), $5-48$.

[HR15] T. Hausel and F. Rodriguez Villegas, Cohomology of large semiprojective hyperkähler varieties, in: De la géometrie algébrique aux formes automorphes (II), Astérisque 370 (2015), 113-156.

[Hir75] H. Hironaka, Triangulations of algebraic sets, in: Algebraic geometry (Arcata, 1974), Proc. Sympos. Pure Math. 29, 165-185. Amer. Math. Soc. (1975).

[HJNTY20] M. Hoyois, J. Jelisiejew, D. Nardin, B. Totaro, and M. Yakerson, The Hilbert scheme of infinite affine space and algebraic K-theory, preprint arXiv:2002.11439 (2020). 
[Iar72] A. Iarrobino, Reducibility of the families of 0 -dimensional schemes on a variety, Invent. Math. 15 (1972), 72-77.

[Jel20] J. Jelisiejew, Pathologies on the Hilbert scheme of points, Invent. Math. 220 (2020), 581-610.

[Kar00] N. Karpenko, Cohomology of relative cellular spaces and of isotropic flag varieties, Algebra i Analiz 12 (2000), 3-69.

[Kol95] J. Kollár, Rational curves on algebraic varieties, Ergebnisse der Mathematik und ihrer Grenzgebiete, Springer-Verlag (1995).

[Kol07] _ Lectures on resolution of singularities, Annals of Mathematics Studies 166. Princeton University Press (2007).

[Mil85] J. Milnor, On the concept of attractor: correction and remarks, Comm. Math. Phys. 102 (1985), 517-519.

[Mor05] F. Morel, The stable $\mathbf{A}^{1}$-connectivity theorems, K-Theory 35 (2005), 1-68.

[MV99] F. Morel and V. Voevodsky, $\mathbf{A}^{1}$-homotopy theory of schemes, Inst. Hautes Étud. Sci. Publ. Math. 90 (1999), 45-143.

[Par96] K. Pardue, Deformation classes of graded modules and maximal Betti numbers, Ill. J. Math. 40 (1996), 564-585.

[Ree95] A. Reeves, The radius of the Hilbert scheme, J. Alg. Geom. 4 (1995), 639-657.

[RS72] C. P. Rourke and B. J. Sanderson, Introduction to piecewise-linear topology, Ergebnisse der Mathematik und ihrer Grenzgebiete, Springer-Verlag (1972).

[Stacks] The Stacks Project Authors, The Stacks Project, http://stacks.math.columbia.edu

[Sum75] H. Sumihiro, Equivariant completion II, J. Math. Kyoto Univ. 15 (1975), 573-605.

[Voe00] V. Voevodsky, Triangulated categories of motives over a field, in: Cycles, transfers, and motivic homology theories, Ann. Math. Stud. 143, 188-238 (2000). 